\title{
EXCITON STATES IN ORGANIC ALLOYS: NAPHTHALENE-PERDEUTERONAPHTHALENE*
}

\author{
Panos ARGYRAKIS, Eric M. MONBERG and Raoul KOPELMAN \\ Department of Chemistry. The Universily of Michigan, Ann Arbor, Mfichigen 78107, USA
}

Received 21 Junc 1975

The lower bandedge energy of the first singlet exciton of nuph thalene has been determined as a function of concentration in a $\mathrm{C}_{10} \mathrm{H}_{8} / \mathrm{C}_{10} \mathrm{D}_{8}$ alloy. Experimentally, the vibronic $0-512$ fluorescence at $2 \mathrm{~K}$ and $1 \mathrm{~cm}^{-1}$ resolution has been measured. Theoretically, the coherent potential approximation (CPA) and the ncgative factor counting (NFC) results have been derived from the work of Hong and Kopelman. Agreement is good within the experimental and computational uncertainties $\left(2-3 \mathrm{~cm}^{-1}\right)$.

Incidentally to an energy transfer study $[1,2]$ we have determined the dependence of an exciton bandedge on concentration in an isotopic mixed crystal of naphthalene/perdeuteronaphthalene. It is of interest to compare these experimental data with present-day theories of excitons in disordered aggregates $[3,4]$. As the first singlet exciton of naphthalene is perhaps the best studied Frenkel exciton system $[1,3,5,6]$ and as our jata have been carefully assembled at $2 \mathrm{~K}$, this study is a stringent test on the reliability of both the available experimental and theoretical techniques. Previous related experimental works are the pioneering photographic studies by Sheka [7] and by Hong and Robinson [8]. These studies were mainly concerned with the 0-0 electronic transition and therefore with the "Davydov components" positions as a function of concentration. The same data were also used in a sim. ilar theoretical analysis by Hoshen and Jortner [9]. This theoretical analysis $[8,9]$ was based on the then available pure crystal parameters like the exciton density-of-states [10] (the early work of Sheka [7] w:s based on the pioneering model of Broude and Rashba [11]). Better experimental parameters have since become available [12-14] as well as more so. phisticated mixed crystal theories [4]. In addition, our study is free of complications due to symmetry restrictions (i.e., free of $k=0$ selection rules) as it

\footnotetext{
* Supported by N1H Grant NS081 16-08 and NSF Grant GH-32578X.
}

concentrates on a band-band transition $[10,15]$ $(0 \rightarrow 512)$. As the lower band (the vibrational exciton band, $512 \mathrm{~cm}^{-1}$ ) has negligible width [16], and due to the very low temperaturc of the experiment $(1.6-1.8 \mathrm{~K})$, we are monitoring not a "Davydov component" (which is ill-defined in the mixed crystal), but the real position of the lower ${ }^{1} \mathrm{~B}_{2 \mathrm{u}}$ exciton band-edge. We compare this position with the theoretical values based both on the coherent potential approximation (CPA) and on the negative factor counting (NFC) method [4] (the latter being based on specific pairwise exciton interactions $[12,17])$.

Experimentally we used higlly purified, isotopic mixed crystals $[1,2]$ of naphthalene $\left(\mathrm{C}_{10} \mathrm{H}_{8} / \mathrm{C}_{10} \mathrm{D}_{8}\right)$. As it became evident that minute amounts of chemical impurities $\left(10^{-3}\right.$ to $10^{-5}$ mole fraction of $\beta$-methylnaphthalene) do not affect the energy of the naphthalene emission, within our precision (even though they significantly affect the intensity), we used data based both on samples doped with such impurity levels as well as "natural samples" (without potassium fusion or zone refining [1]). The 24002900 A excitation was from a xenon lampappropriately filtered $[1,2]$, at $1.6-1.8 \mathrm{~K}$. The emission was monitored with a $1 \mathrm{~cm}^{-1}$ resolution by a double grating spectrometer with digital photon counting and simultaneous celibration (interphused with an IBM 360/67 computer, enabling calibration, smoothing and differential integration on a Graphics terminal) $[1,18]$. An example of an individual spec- 


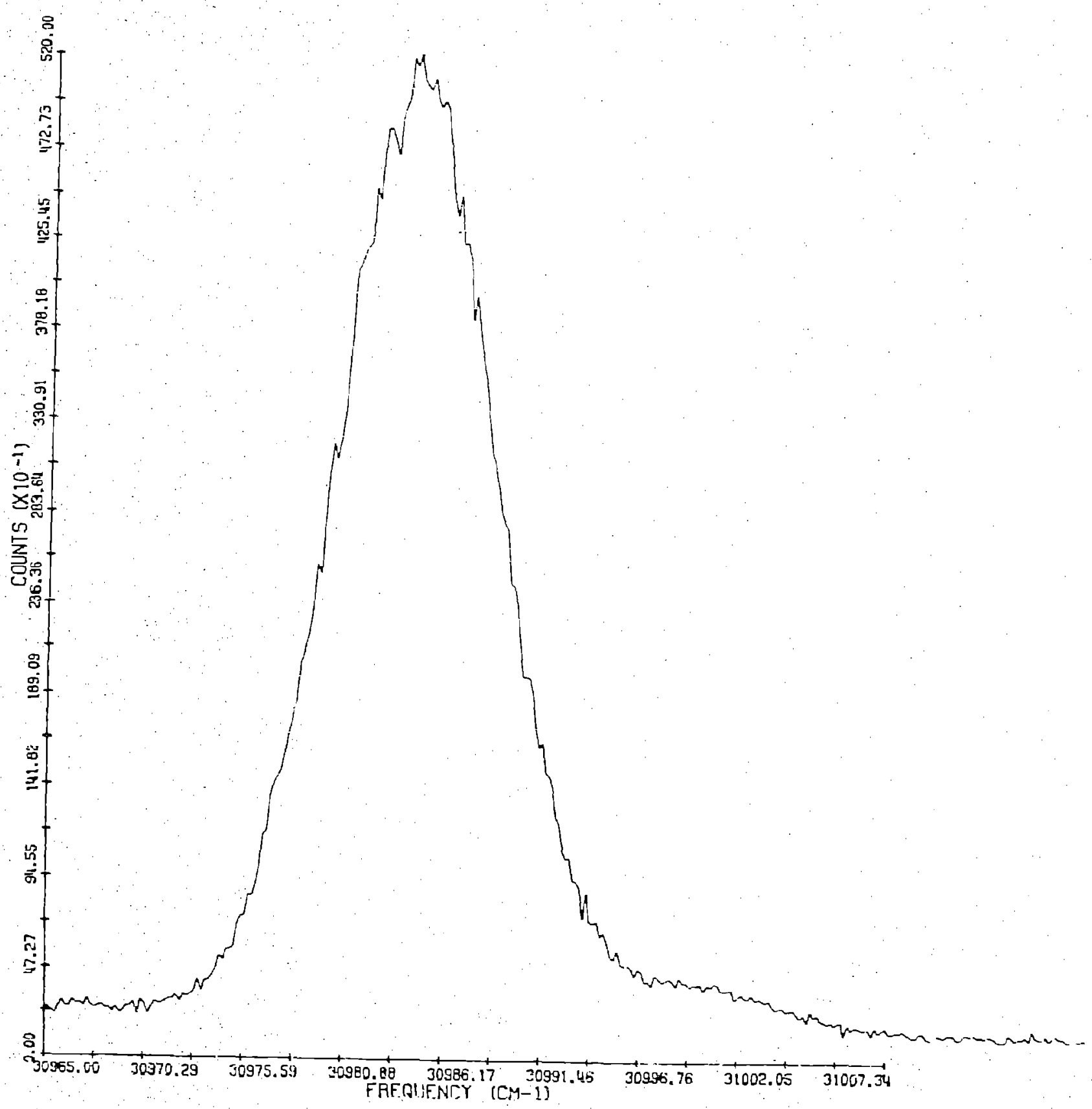

Fig. 1. A sample $0 \rightarrow 512$ fluorescence of the naphthalene ${ }^{1} \mathrm{~B}_{2 \mathrm{u}}$ exciton in the $\mathrm{C}_{10} \mathrm{H}_{8} / \mathrm{C}_{10} \mathrm{D}_{8}$ "alloy". The mole fraction ( $C_{\mathrm{A}}$ ) of $\mathrm{C}_{10} \mathrm{H}_{8}$ is 0.35 , the temperature $2 \mathrm{~K}$ and the resolution $\mathrm{i} \mathrm{cm}^{-1}$. The intensity is in photon counts, the energy in cm-1. 
trum is given in fig. 1 . The experimental peak-heights are summarized in fig. 2, together with a theoretical curve. We omit the points at medium dilution of $\mathrm{C}_{10} \mathrm{H}_{8}$, as at these concentrations each spectrum contains several peaks, due to various clusters (monomer, dimers, etc. [1]).

The results (fig. 2) are very satisfactory. We note that we have an experimental error in measuring the peak of about $1 \mathrm{~cm}^{-1}$. In addition we have an uncertainty related to the " 512 ". $\mathrm{cm}^{-1}$ vibration. The vibrational frequency (actually measured to be about 509 $\mathrm{cm}^{-1}$ in the pure crystal) might slightly change with concentration - up to $1-2 \mathrm{~cm}^{-1}$. In addition there is really another accidentally degenerate vibration involved $[1,16,19]$. All this leads to an experimental error of about $2-3 \mathrm{~cm}^{-1}$. We notice that the theoreti. cal data have computational uncertainties, the CPA method of about $2 \mathrm{~cm}^{-1}$ and the NFC of about 2.5 for some points and $5 \mathrm{~cm}^{-1}$ for others [4]. This is apart from errors inherent in the rnodel and in the pure

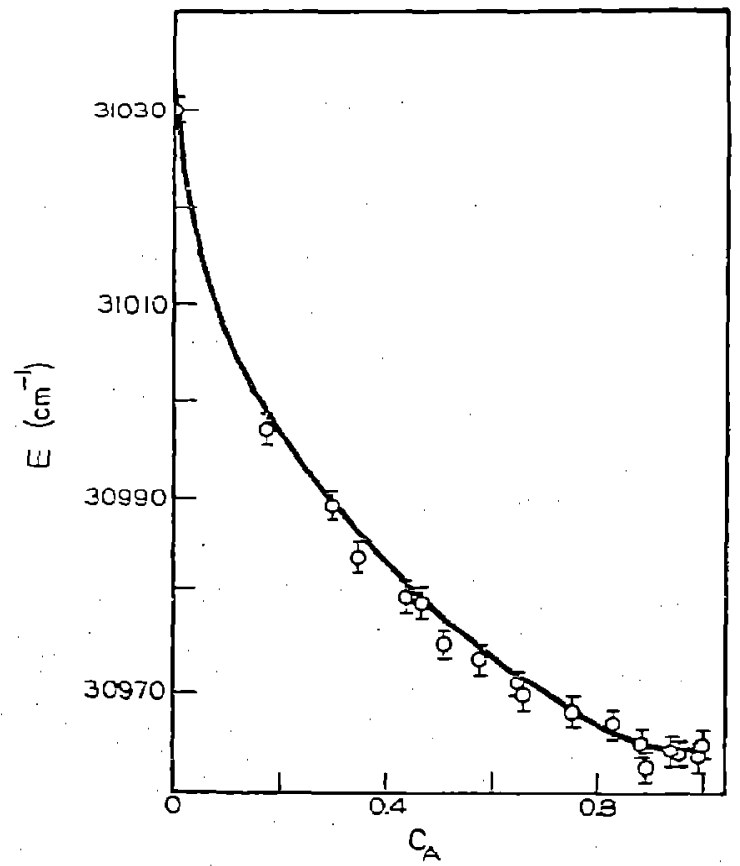

Fig. 2. Experimental and calculated bandedge energies of the maphthalene ${ }^{1} B_{2 u}$ exciton as function of concentration in a $C_{10} H_{3} / C_{10} D_{8}$ "alloy". $C_{A}$ is the mole fraction of $C_{10} H_{8}$. The error bars of the experimental points (the three lowest concentration points are taken from ref. (1]) are discussed in the text, as is the uncertainty in the theoretically computed curve (solid line). crystal data used as input. Even though we constrained the theoretical band-edge value at $C_{\mathrm{A}}=1$ to coincide with the experimental one, emphasizing that this test is on the mixed crystal interpolation formulae, the abcve mentioned theoretical uncertainties are still pertinent. We therefore consider the fig. 2 results to indicate very good agreement between theory and experiment. We also noted above that we have avoided comparing experiments to theory in concentration regions well below the percolation concentration $[2,4]$. While we do not expect the CPA theory to work at such low concentrations, the NFC method should not be affected. However, the band-edge concept breaks down, due to clustering. (We reserve our investigations in this region for a future publication.) Unfortunately, due to computation costs, the computer band-edge results of the NFC method [4] are, within their precision, identical with the less valid CPA values. Therefore we cannot presently make a distinction between these two theoretical methods. However, we feel that a computational refinement is unjustified in view of the experimental uncertainties. As to the experimental points being mostly $1-2 \mathrm{~cm}^{-1}$ below the theoretical curve, theie may be a systematic error due to a slight shift or asymmetric contraction of the vibrational and/or electronic exciton band with concentration change, but this discrepancy is well within the combined experimental and computational uncertainties.

In summary, we compare experimental and theoretical data on the naphthalene ${ }^{1} B_{24}$ exciton bandedge in isotopic mixed crystals $\left(\mathrm{C}_{10} \mathrm{H}_{8} / \mathrm{C}_{10} \mathrm{D}_{8}\right)$. Both experimental and theoretical values are more refined than in previous studies. The good agreement we find provides us with increased confidence in both our experimental and theoretical approaches to the problems of excitons in molecular alloys.

\section{References}

[1] F.W. Ochs, Ph. D. Thesis, The University of Mishigan (1974).

[2] R. Kopelman, E.M. Monberg, F.W. Ochs and P.N. Prasad, Phys. Rev. Letters 34 (1975) 1506: unpublished data.

[3] R. Kopelman, in: Excited states, Vol. 2, ed. E. Lim (Acadernic Press, New York, 1975).

[4] H.-K. Hong and R. Kopelman, J. Chem. Phys. 55 (1971) 5380. 
[5] E.I. Rashba and E.F. Shelia, in: Physics of impurity centres in crystals, ed. G.S. Zavt (Acad. Sci. Estonian SSR, Tallin, 1972).

[6] F.W. Ochs, P.N. Prasad and R. Kopelman, Chem. Phys. 6 (1974) 253.

[7] E.F. Sheka, Opt. i Spekiroskopiya 10 (1961) 684 [English transl. Opt. Spectry. 10 (1961) 360) ; Bull. Acad: Sci. USSR Phys. Ser. 27 (1963) 501.

[8] H.-K. Hong and G.W: Rohinson, J. Chem. Phys. 52 (i 970$) 825 ; 54(1971) 1369$.

[9] J. Hoshen and J. Jortner, J. Chem. Phys. 56 (1972) 5550.

[10] S.D. Colson, D.M. Hanson, R. Kopalman and G.W. Robinson, J. Chem. Phys. 48 (1968) 2215.

[11] V.L. Broude and E.I. Rasliod, Fiz. Tvird. Tela 3 (1961)
1941 [English transl. Soviet Phys. Solid State 3 (1962) $1415]$.

[12] H.-K. Hong and R. Kopelman, J. Chem. Phys. 55 (1971) 724.

[13] H.-K. Hong and R. Kopelman, J. Chem. Phys. 57 (1972) 3888

[14] F.W. Ochs and R. Kopelman, unpublished.

I 15 I S.D. Colson, R. Kopelman and G.W. Robinson, J. Chem. Phys. 47 (1967) 27. 5462.

[ 6] D.M. Hanson and A.R. Gee, J. Chem. Phys. 51 (1969) 5082.

[17] D.M. Hanson, J. Chem. Phys. 52 (1970) 3409.

[18] [.W. Ochs and R. Kopelman, unpublished.

[19] D.M. Hanson, J. Chem. Phys. 51 (1969) 5063. 